\title{
Time for Change? The Why, What and How of Promoting Innovation to Tackle Rare Diseases - Is It Time to Update the EU's Orphan Regulation? And if so, What Should be Changed?
}

\author{
Denis Horgan $^{a}$ Barbara Moss ${ }^{\text {b, c }}$ Stefania Bocciad, e \\ Maurizio Genuardif, g Maciej Gajewski ${ }^{\text {h }}$ Gabriele Capurso ${ }^{i}$ \\ Pierre Fenaux ${ }^{j, k}$ Beatrice Gulbis ${ }^{k, l} \quad$ Mariangela Pellegrini ${ }^{k}, m$ \\ Maria del Mar Mañú Pereirak, $n$ Victoria Gutiérrez Valle ${ }^{k, n}$ \\ Iñaki Gutiérrez Ibarluzea ${ }^{0} \mathrm{p} \quad$ Alastair Kent $^{\mathrm{q}}$ Ivana Cattaneo ${ }^{\mathrm{r}}$ \\ Beata Jagielska $^{\mathrm{s}}$ Ivica Belinat ${ }^{\mathrm{t}}$ Birute Tumiene $^{\mathrm{u}}$ Adrian Ward $^{\mathrm{v}}$ \\ Marisa Papalucaw
}

\begin{abstract}
a European Alliance for Personalised Medicine, Brussels, Belgium; ${ }^{b}$ Europacolon (UK), London, UK; ${ }^{\mathrm{C} B o w e l}$ Cancer UK, London, UK; ${ }^{\mathrm{d}}$ Sezione di Igiene, Dipartimento Universitario Scienze della Vita e Sanità Pubblica, Università Cattolica del Sacro Cuore, Rome, Italy; e Department of Woman and Child Health and Public Health - Public Health Area,

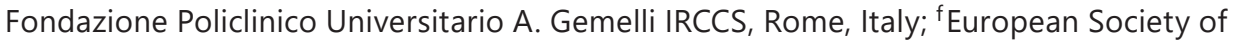
Human Genetics, Vienna, Austria; ${ }^{9}$ Complex Operational Unit, Medical Genetics, Fondazione Policlinico Universitario A. Gemelli IRCCS, Rome, Italy; ${ }^{\text {h}}$ Alexion, Zurich, Switzerland; iPancreatoBiliary Endoscopy and EUS Division, Pancreas Translational and Clinical Research Center, San Raffaele Scientific Institute IRCCS, Milan, Italy; ${ }^{j}$ Service d'hématologie seniors, Hôpital St Louis / Université Paris 7, Paris, France; ${ }^{k}$ ERN-EuroBloodNet, the European Reference Network on Rare Hematological Disease, Paris, France; 'Ht. ERASME-CUB, Brussels, LHUB-ULB, Brussels, Belgium; mm Assistance Publique-Hôpitaux de Paris, Hôpital Saint-Louis, Paris, France; ' 'University Hospital Vall d'Hebron - Vall d'Hebron Research Institute, Barcelona, Spain; ${ }^{\circ}$ EuroScan International Network, Cologne, Germany; ${ }^{\mathrm{P} B I O E F}$, Basque Foundation for Health Innovation and Research, Barakaldo, Basque Country, Spain; 9Independent Patient Advocate, London, UK; ${ }^{\mathrm{r}}$ Novartis Pharma SpA, Origgio, Italy; ${ }^{\mathrm{s}} \mathrm{Clinic}$ of Oncological Diagnosis and Cardio-oncology, Maria Skłodowska-Curie Institute of Oncology, Warsaw, Poland; ${ }^{\mathrm{t}}$ Coalition of Healthcare Association, Zagreb, Croatia; "Vilnius University Hospital Santaros Clinics, Vilnius, Lithuania; 'F. Hoffmann-La Roche Ltd, Basel, Switzerland; w'Imperial College London, London, UK
\end{abstract}




\section{What Is It about?}

Rare diseases is a priority area for EU Community action within the framework for action in the field of public health. A conducive regulatory environment can further support the development of medicines to treat rare diseases. Overall there is a need for joined-up regulatory process coordination. Better integration of regulatory pathways and better integration of regulatory systems, such as scientific tools and methods to generate evidence, would be helpful. This paper explores the successes - and imperfections - of the regulation, and sets out some options for reflection on remedying imperfections within the broader framework of improving patient access.

\section{Keywords}

Rare disease $\cdot$ Regulatory $\cdot$ Member states · Orphan regulation · European Commission · Patients · Personalised healthcare - Incentives · Challenges · Access · Biosimilars - Unmet need $\cdot$ Reimbursement · Diagnostics · Biomarkers · Inequality $\cdot$ Treatment · Data · Ultra-rare disease $\cdot$ Empowerment $\cdot$ Patient $\cdot$ Citizens $\cdot$ Access

\section{Abstract}

Since developments are global in the healthcare arena, more should be done to align EU and other big markets' regulatory practices for rare disease patients. Notwithstanding efforts and cooperation between the US and EU aimed to harmonize their strategic plans in the field of orphan drugs, regulatory criteria and procedures to gain the designation, terms and classifications should be still harmonised. Aligning the criteria of prevalence and support to orphan medicines in the various jurisdictions internationally, would facilitate patient recruitment eventually at global level, so as to gain the data and the biological insights required to identify biomarkers and appropriate endpoints needed for progressing clinical development. A conducive regulatory environment can further support the development of medicines to treat rare diseases. Overall there is a need for joined-up regulatory process coordination. Better integration of regulatory pathways and better integration of regulatory systems, such as scientific tools and methods to generate evidence, would be helpful. There is a need to revise and agree the current frameworks to be improved which will take into account the considerations and challenges to diagnose and treat different rare diseases and improve quality of life. Deliberative processes with multi-stakeholders' involvement for reimbursement should be considered. This paper explores the successes and limitation of both the regulation and its implementation mechanisms in the current regulatory context, and suggests some improvements that could maximise its benefits and boost rare disease research even further.

\section{Introduction}

The European Union's (EU) Regulation (EC) No. 141/2000 on orphan medicinal products (OMPs) (referred to as "the regulation" in this paper) states that "patients suffering from rare conditions should be entitled to the same quality of treatment as other patients," and concludes that "it is therefore necessary to stimulate the research, development and bringing to the market of appropriate medications by the pharmaceutical industry" [1]. Rare diseases had already been identified as a priority area for Community action within the framework for action in the field of public health [2], and the regulation's stated aim is - "to provide incentives for the research, development and placing on the market of designated orphan medicinal 
Horgan et al.: Time for Change? The Why, What and How of Promoting Innovation to Tackle Rare Diseases

products." It set up a mechanism to ensure that "orphan medicinal products eligible for incentives should be easily and unequivocally identified," with the condition that "objective criteria for designation should be established" [3]. The core incentive of the regulation is the granting of 10 years ( +2 years for paediatric orphan medicines) of marketing exclusivity and a range of financial and scientific provisions granted via the European Medicines Agency to support product development and application for Marketing Authorisation.

Nearly two decades later, the success of the measure has been demonstrated. Investment both from public research funders and from companies of all sizes in rare disease research has resulted in the approval of more than 150 orphan drugs - compared with just eight therapies for rare diseases available before the adoption of the regulation. That translates into a lot of patient benefit. With clinical research stimulated by the legislation, the EU sees some 2,000 clinical trials providing still more innovation or hope for treatments in the current R\&D pipeline [4].

But over the intervening years, the limitations in the functioning of the legislation have become apparent too, and these merit attention if the beneficial effects for patients and caregivers are to be maximised [5]. This paper explores the successes and limitation of both the regulation and its implementation mechanisms in the current regulatory context, and suggests some improvements that could maximise its benefits and boost rare disease research even further.

The discussion needs to be precise if it is to be effective. Review of the functioning of the regulation may coincide with a period of more intense scrutiny and concerns over containing the rise of expenditure to ensure sustainability of healthcare systems, with a particular focus on expensive innovation which are often developed within the orphan conditions. While there is undoubted importance in the wider but distinct debate over healthcare costs, it does not bear directly on reviewing the orphan medicines regulation [6].

At the same time, economic questions do, however, have relevance to the debate on orphans, since patients' access to the medicines that become available is conditioned by the national arrangements for reimbursement or listing of products: there is an increasing tension between the potential access to agents that can modify or even cure rare diseases, and the models for reimbursement available to European payers. Part of this hesitancy can be ascribed to the novelty of the challenges presented by many innovative treatments, which by their nature present unknowns to payers. Clearly, there is also a need to deal with uncertainty with regard to value demonstration, especially when value or values are perceived not to be sufficiently demonstrated. The risk is that such powerful economic reservations can have a cumulative negative impact on the motivation for pursuing research into rare disease treatments - thus running counter to the guiding principle of the legislation itself [7]. Current value assessment rules across Europe for orphan drugs remain largely inadequate and can become a real fourth hurdle to effective patient access to those treatments [8].

The regulation's stimulation of new product development has also helped promote the development of EU biotech companies. The last two decades have witnessed the emergence of more than 150 small and medium enterprises (SMEs) focusing on rare diseases. No wonder that one of the prominent Members of the European Parliament over this period, Francoise Grossetête, emphasised the importance of the regulation in addressing "real medical needs" and generating "therapeutic breakthroughs" [9]. The underlying strength of the concept of providing incentives for R\&D in areas of unmet need is confirmed by the fact that Germany and other Member States are now exploring whether OMP-type incentives could contribute to solving the major risks of antimicrobial resistance (AMR), through promoting development of new anti-bacterials even where simple market economics do not provide sufficient motivation for investment [10].

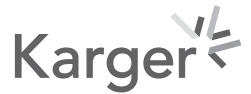


Horgan et al.: Time for Change? The Why, What and How of Promoting Innovation to Tackle Rare Diseases

Thanks to increased investments and the associated efforts thus made possible, some rare diseases now benefit from effective treatments. There are leading examples in the area of haemophilia, paroxysmal nocturnal haemoglobinuria (PNH), and some lysosomal storage diseases such as Gaucher. The full list of conditions for which "orphans medicines" have been launched in Europe is too extensive to reproduce here, but by way of illustration it ranges from rare cancers to rare variants of common diseases (pulmonary hypertension, neonatal diabetes) and to rare congenital, mostly childhood-onset disorders (Gaucher, cystinosis, inherited hyperammonaemias) [11].

However, these tales of success should not lead to any delusions that the process has been - or is becoming - easy. Successes in developing innovative treatments are hard-won. Without consistent and determined effort, innovation does not happen - and innovation in rare diseases is all the more challenging. The key elements of the innovation process are well documented, but the nature of the challenges is perhaps not always fully appreciated by those outside the healthcare sector, being seen as costs and not as investments. Rare diseases are categorized as "orphan diseases" because their occurrence in a small number of patients means that, despite apparent high unmet medical need, there is limited scientific understanding, making it difficult to justify the development risk and investment to develop new treatments. The OMP regulation was developed explicitly to support efforts in this field of innovation [12].

The positive results, though impressive, still represent only a small part of the huge need for tackling the thousands of rare diseases that remains unmet. Estimates of the number of affected individuals vary in detail, but suggest as many as 446 million worldwide, and 30 million in Europe. These individuals face challenges, including delays and errors in diagnosis, a lack of clinical guidelines and care pathways, and limited information and support, all of which considerably affects their lives and their families or caregivers. An estimated $5 \%$ of rare diseases currently have a treatment option, which leaves $95 \%$ of rare diseases without. And limited availability is strikingly evident in some therapeutic areas and for genetic rare diseases. $70 \%$ of rare diseases are onset in childhood, and around 3 out of 10 of these children die before reaching 5 years of age [13]. As the German association of researched based pharmaceutical companies (VFA) says, the well-identified challenge of orphan diseases - it estimates up to 8,000 - "will need the incentives provided in the Orphan Regulation for many more years" [14]. At the World Orphan Drug Conference in November 2019, opening speakers agreed that the regulation had incentivised drug development, but pointed out that only $1 \%$ of rare disease therapies receiving authorisation are actually marketed, demonstrating that obstacles to access need urgent fixing [15].

A significant number of rare diseases failed to attract R\&D investment and remain orphan, and reflection is needed on how the support should evolve to strengthen the development of appropriate treatments for them. Paediatric cancers offer a significant example of both the limitations of the current systems and the need to develop new strategies for the treatment of rare diseases. Cancer is the first cause of death by disease for children over 1 year of age in Europe. Yet drug companies have demonstrated less interest in paediatric cancers, since this has been perceived as an unattractive commercial market [16]. As a consequence of insufficient motivation or incentivisation, more that $50 \%$ of drugs given to children have never been investigated in this population, but only in adults, and those drugs are administered "offlabel". By contrast, 2,000 candidates for innovative medicines have been developed for adult oncology and billions of dollars invested by industry in continuing research [17].

So despite the wider successes of the orphan medicines regulation, very limited benefit has accrued to children with cancer: in the last 10 years, they have been able to receive only two new adult drugs authorized with a specific paediatric indication in oncology. And in the last 16 years, only 15 orphan drug designations have been granted in this field, and only two

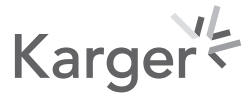


orphan medicines have been approved for the treatment of a malignancy occurring specifically in children. Moreover, $68 \%$ of the 31 anti-cancer medicines with orphan drug designation that were approved for the treatment of a cancer occurring both in children and in adults still have no information for paediatric use in their Summary of Product Characteristics at a median of 7 years after their first marketing authorisation [18].

In the EU, an OMP will lose its designation automatically after 10 years (sometimes 12 provided that a paediatric development has been successfully completed within these 10 years) [19]. The designation may also be withdrawn voluntarily. As a result, tracking how many products are currently authorised to treat rare diseases is difficult. At the end of 2018, a total of 164 orphan-designated medicines had been granted a marketing authorisation, but only 107 currently have active marketing authorisation in the European Union register. The remaining 57 either have had their orphan status withdrawn or their market exclusivity has expired. These medicines are still used to treat a rare disease but they are not recognised as such anymore. Zamora et al. [20], found that one of the intended effects of the regulation, to grant equal availability to OMPs to patients in the EU, was only partially achieved, with important variations in availability and access. That paper notes the negative impact of different regulatory frameworks for the development of orphan drugs, with separate governance, multiple assessments, and varying approaches and priorities to unmet medical needs in the public health systems.

Market exclusivity, one of the central features of the regulation, provides for the protection during a limited period from competition from similar medicines for the same indication. This is an important incentive for development of medicines for rare diseases [21]. However, its operation is not always fully effective. In particular, difficulties can arise when a company develops further indications for a product (encompassed under the umbrella term of the defined orphan condition). Market exclusivity also faces a challenge of enforceability at Member State level, which discourages continued investments to develop and launch new indications for an existing orphan drug. The value of multi-indication approach in rare diseases is that every new indication is a completely new disease, which in most cases, lacks authorized treatments. This is significantly different from the situation in more prevalent diseases, where companies often expand their indications to enable treatment of less severe patients suffering from the same original condition. This difference extends to the impossibility of making virtually simultaneous multi-indication launches, and underlines the inevitability of staggered introduction - since it can take 10-15 years to develop all possible indications.

The challenges are intensified by different value assessment frameworks from country to country, often with a focus on short-term budgetary considerations or simple cost-effectiveness methods. Germany's relatively congenial route for orphan drugs, directly linking value assessment to significant benefit and establishing a carve-out for orphan products, contrasts with the UK's less successful approach in which its highly specialised technologies pathway still employs cost-effectiveness thresholds, consequently disqualifying many orphan drugs. These issues, however, lie at the interface of authorisation and reimbursement procedures, and merit exploration at more depth in a further paper [22].

\section{What Sort of Improvement?}

The aim of the OMP Regulation is that "patients suffering from rare conditions should be entitled to the same quality of treatment as other patients" [23]. If it has not yet delivered on its aims, does that mean that the regulation is misguided and misconceived? That would hardly be a conclusion consistent with the evidence, given that in the relatively short (in the context of drug development timetables) period of its existence it has in fact led to the generation of a large number of effective new therapies. Grossetête pointed out that the continuing

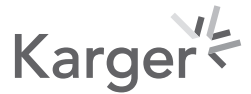


Horgan et al.: Time for Change? The Why, What and How of Promoting Innovation to Tackle Rare Diseases

existence of patient need "does not mean that the orphan regulation is not delivering; it merely underlines that R\&D in this area is highly complex and that regulations are not the only solution." She warned against over-reactions that could undermine the existing regulation and counselled careful assessment of regulatory barriers. "Issues related to patient access will not be solved by a piece of legislation alone," she said [24].

Completing the R\&D process remains a challenge for an orphan medical product: there are issues relating to the rarity of the conditions, the variable phenotype, the limited understanding of their biological mechanisms, the difficulties related to diagnosis, endpoints acceptance, and recruitment across many countries. In light of the many resulting difficulties in generating adequate evidence, it is not surprising that about $30 \%$ of orphan designations granted in Europe failed their development, with drugs abandoned during the development or where marketing authorisation was refused or the application withdrawn [25]. A study conducted in 2018 by European Union regulators reviewed 157 orphan-designated medicines registered between 2000 and 2013 and found that orphan medicines have a more complex clinical development and lower approval and success rate compared with nonorphan medicines [26]. A further challenge is that at the time of submitting the application for a marketing authorisation, data may be required to demonstrate significant benefit to maintain the orphan status of the product.

It remains the case, as it was in 2000 , that it is "necessary to stimulate the research, development and bringing to the market of appropriate medications by the pharmaceutical industry" [27]. And again, in the spirit and letter of the regulation, this means it is still necessary "to provide incentives" for the process, through mechanisms under which "orphan medicinal products eligible for incentives should be easily and unequivocally identified," and on the basis of "objective criteria." If the aim is to be pursued, what may be appropriate is to review the operation of the regulation two decades on, in light of imperfections that have been identified as inimical to its objectives, and in light also of the changes - often dramatic - that have occurred in the underlying science and technology and that have opened up new dimensions to the understanding of disease and of therapy.

\section{What to Avoid?}

Limiting incentives for OMPs would only be justified if there was no unmet medical need in the field of rare diseases - which is manifestly not the case. The optimal outcome to meeting unmet need is for improvements in the regulation through rigorous exploration of its impact on research and development. The European Society of Paediatric Oncology (ESPO) stressed the need for "a high level of market protection to facilitate company investment." The European Commission pointed out in the report of its own stakeholder conference on the OMP and the paediatric regulation in June 2019 that "the ultimate objective... was to increase therapeutic choices for patients suffering from rare diseases and children by bringing more medicinal products for those therapeutic areas to the market." To improve the development of and access to treatments for patients with rare diseases is the focus of discussions [28]. It has been the consistent claim of the pharmaceutical industry and of many research institutes that without effective incentives the development of new treatments for rare diseases will slow down.

Other questions about amendments to the rules might relate to the definition of an orphan condition - of unmet medical need, of significant benefit, and particularly where the underlying causes are complex and many biomarkers drive the clinical course and change the nature of the condition. The "orphanisation" of common diseases, especially cancers, based on markers that distinguish many sub-populations in a given disease, suggests a need for discussions and dialogue on the line between personalized and orphan medicines.

Questions might relate to the relevance of prevalence in different circumstances. The European Union defines a rare (or "orphan") disease as a life-threatening or chronically debil-

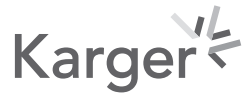


Horgan et al.: Time for Change? The Why, What and How of Promoting Innovation to Tackle Rare Diseases

itating disorder that affects $<5$ in 10,000 people in the European Union. However, the prevalence can be much lower, leading to the concept of the "ultra-orphan disease" for diseases with an estimated prevalence of $<1$ in 50,000 people [29]. Such low prevalence, together with the limited available scientific knowledge, explains the challenges sponsors face when developing orphan medicines (e.g., lack of relevant and agreed endpoints, limited sample size, costly processes, ...).

Gene/cell therapies, genomics, digital biomarkers in combination with therapeutic options open new opportunities for many rare diseases, but also uncertainties, and might require quite distinct (and possibly wider reaching) regulatory approaches not encompassed by the current OMP Regulation, with the objective of gathering as much informative data as possible from clinical use. At the same time, modern collaborations and data analyses (e.g. AI driven) come with potential impact on market exclusivity. And questions relate to both the definitions and the data required for demonstration of benefit and value.

To facilitate progress in the fundamental knowledge of rare diseases and alleviate the roadblock in clinical development of orphan medicines, cooperation of a global and longitudinal nature, from designation to market, should be envisaged among regulatory, authorities, patients organisations, academia and developers of both medicines and support technologies, and payers. New rules of the game (for IP and data protection, for confidential data sharing) and new standards (shared across the stakeholders) will have to be developed.

The number of medicines that receive a marketing authorization will depend on the generation of sufficient and acceptable evidence to support their authorization. This process can take as long as for a non-orphan product; orphan-designated medicines are also subject to similar attrition rates as non-orphan medicines during development. When an orphandesignated medicine is eventually submitted for a marketing authorization, it must show that it continues to meet the criteria for orphan designation, such as its significant clinical benefit compared with other available treatment options at that time [30]. Orphan status maintenance is indeed a prerequisite to the market exclusivity incentive. It is not uncommon for medicines that were initially orphan designated to "lose" their orphan status at this point, despite completing the lengthy research, development, and authorization processes and procedures. Recent court cases highlight this issue and the strict approach of the European Medicines Agency (EMA) and European Union Commission in this regard [31]. Different levels of incentives might be applied to different circumstances, and exceptions provided for in the case of specific diseases [32].

For some rare diseases, therapies are available or at promising stages of development. At the same time, there are many rare and orphan diseases without much R\&D activity and in those areas incentives are critically needed. It should be assessed if the current incentives are strong enough and whether some prioritisation is required [33].

Legislation could envisage close and early collaboration among regulators, patients and payers in defining the value of a product under development, and should reduce the uncertainty surrounding revenue on investment and mitigate the effects of quasi-simultaneous development of orphan medicines by different sponsors. Reflection might extend to whether OMP changes should aim to strengthen paediatric research, and whether the paediatric aspect has been sufficiently taken account of in the regulation. For instance, a specific opinion might be required on the development plan (as is the case with the EMA Paediatric Committee opinion for paediatric medicines), or adherence to the proposed plan be verified (analogous to the "compliance check" for paediatric medicines development) [34].

There are real challenges to be overcome in these areas that could improve the prospects of successful development of orphan drugs. But any change to the OMP scheme should relate to improving achievement of its stated purpose.

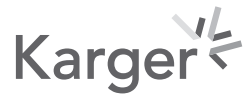


Horgan et al.: Time for Change? The Why, What and How of Promoting Innovation to Tackle Rare Diseases

\section{Extrapolation of Biosimilar Indications}

A particular issue has arisen with the emergence of biosimilars, where fine-tuning of regulation could help to promote innovation - and where failure to do so could have the opposite effect. There are merits to the development and wider use of biosimilars. Coming to the market only once patents have expired, alongside originator medicines they provide a balance between supporting innovation in biologics and protecting the economic sustainability of healthcare systems, providing therapeutic progress and thus better treatment for patients on the one hand, and on the other hand assuring greater affordability of medicines through competition after loss of protection rights. Some Member States even claim that over and above their savings potential, biosimilars also incentivize originator companies to develop new medicines to generate business instead of relying on revenues from "old", no longer protected medicines.

Where the fine-tuning is required relates to guaranteeing the protection that the EU legislative framework provides for innovations. In principle, the system offers protection but the protection is linked to individual indications, and in practice this indication-specific character of the protection is not always respected. Failure to respect the principle of single indications can lead to reducing rather than increasing the treatment options for patients. However, the consequences for other pathologies should be carefully analysed, especially in the case of new cancer treatments which their indication is no longer location based but biomarker based.

The difficulty arises where one compound has the ability to treat several diseases or conditions, some rare, some not. As soon as a biosimilar becomes available with an authorization for a first indication in a condition for which no protection exists, its wider use is frequently advocated, including for other indications which still enjoy protection. This not only violates the balance the system is designed to maintain. It also results in harm for innovators, stifling their interest in innovation. Not only are the incentives for research beyond a first indication for a compound de facto extinguished, but R\&D in that area is actively discouraged. Allowing, or worse still promoting, such practice increases the risk that patients - particularly those suffering from rare diseases - will continue to lack a treatment option in a world of drugs developed on the principle of "one compound - one indication." This principle should be at least reconsidered [35].

The remedy might emerge from achieving an operational consensus on points such as:

- The facilitation of biosimilar use should respect the approved therapeutic indications

- Use beyond approved indications or additional indications should not be encouraged solely for economic reasons but therapeutic ones

- Standards for regulatory approval - including the extrapolation of indications, postmarketing surveillance and risk-management plans - should be the same for reference biologics and biosimilar.

\section{Conclusion}

It may be concluded that the regulation has been - and largely remains - valuable, but that it merits some considerations to fully exploit its potential. It is also argued that improvements should focus on the initial objective of the regulation - promoting the development of therapies for rare diseases.

There are many economic issues that merit further discussion - but this paper is not the place for them. Most obviously, the interplay of market authorisation and reimbursement processes can complicate the operation of the incentives under the Orphan Medicines Regu-

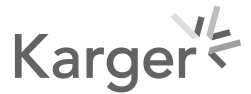


lation and the Paediatric Regulation - such as where payers are tempted to impose off-label use of a cheaper alternative to a product covered by market exclusivity.

There are undeniable tensions and misunderstandings over economic issues between the pharmaceutical industry and public stakeholders that would benefit from dialogue and a greater sense of mutual understanding. The public perception of high returns on investment fuels public concerns over what is seen to be opacity over development costs - often intensified by open questions over the quality of supporting data for authorization applications and how far real-world evidence can or should be adduced. At the same time, industry is faced with persistent inconsistencies in national procedures that it sees as causing delays and imposing unnecessary costs.

The establishment of a climate of trust would also make it possible to advance further in the definition of eligibility criteria for incentive, where open dialogue among all stakeholders is increasingly necessary - something all the more appropriate as patient interests are becoming recognised as the legitimate and indispensable focus for all areas of therapeutic development. Unquestionably, metrics to define value and outcomes can be usefully improved by such improved understanding. These complex issues will be addressed in subsequent papers.

There are real challenges relating to the effective implementation of the OMP Regulation, and of the paediatric incentives scheme. As PHARMIG has stated: "The objective of the evaluation of both regulations should be to ensure the objectives of these regulations, namely to increase the therapeutic choices for children and patients suffering from rare diseases" [36].

But the discussion of changes should not attempt to resolve broader questions of the overall funding of healthcare systems. Re-opening of the regulation may be timely and necessary, but not because of concerns over the cost of innovative treatments for rare diseases. The incentive framework for orphan drugs should be strengthened and improved to promote further innovation, especially in areas that have not so far received adequate incentives. In this context, it should be borne in mind that re-opening the regulation is not without risks: if the discussion is not managed carefully, it could have unintended consequences in discouraging rather than encouraging innovation. It will be the responsibility of the European Commission to provide a constructive framework to steer the discussion.

\section{Acknowledgements}

We would like to thank the members of the European Alliance for Personalised Medicine, and thanks to the contribution from the consortium of DigitalHealthEurope: Support to a Digital Health and Care Innovation initiative in the context of Digital Single Market strategy.

\section{Statement of Ethics}

This work was prepared and approved by a multi-stakeholder group of authors which included amongst others representatives from patient organisations and scientific societies.

\section{Conflict of Interest Statement}

All authors declare that they have no competing interests.

Ivana Cattaneo is a Novartis employee and has stock options in Novartis.

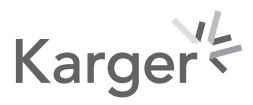


Horgan et al.: Time for Change? The Why, What and How of Promoting Innovation to Tackle Rare Diseases

\section{Author Contributions}

D.H. conceived, built and drafted the article with critical input from his co-authors and experts from within the membership of the European Alliance for Personalised Medicine.

\section{Funding Sources}

This work was supported by the in-kind support of the members of the European Alliance for Personalised Medicine.

\section{References}

1 European Parliament and the Council of the European Union. Regulation (EC) No 141/2000 of the European Parliament and of the Council of 16 December 1999 on orphan medicinal products. Official J Eur Communities. 2000;43:L18.

2 Rare Diseases: A Public Health Priority at http://ec.europa.eu/health/ph_threats/non_com/rare_diseases_en.htm.

3 European Parliament and the Council of the European Union. Regulation (EC) No 141/2000 of the European Parliament and of the Council of 16 December 1999 on orphan medicinal products. Official J Eur Communities. 2000;43:L18.

4 Friends of Europe, Event Report, Equal Access to care for rare disease. Summer 2019.

5 Director-General for Research and Innovation (European Commission). Science, Research and innovation performance of the EU. 2018. ISBN 978-92-79-69744-9.

6 Commission Working Staff Document on the experience acquired as a result of the application of Regulation (EC) No 141/2000 on orphan medicinal products and account of the public health benefits obtained at http:// ec.europa.eu/enterprise/pharmaceuticals/orphanmp/doc/2006/summarycomments.pdf.

7 Zamora B, Maignen F, O’Neill P, Mestre-Ferrandiz J, Garau M. Comparing access to orphan medicinal products in Europe. Orphanet J Rare Dis. 2019 May;14(1): 95.

8 Hampson E, Taylor K, Sanghera A. Patient access to innovative medicines in Europe - A collaborative and value based approach. Deloitte Centre for Health Solutions; January 2019.

9 European Commission Report Conference "Medicines for Rare Diseases and Children: Learning from the Past, Looking to the Future". Brussels, June 2019.

10 European Commission, 2016. Inventory of Union and Member State incentives to support research into, and the development and availability of, orphan medicinal products. State of play 2015.

11 List of medicinal products for rare diseases in Europe, Orphanet Report Series, January 2020. https://www orpha.net/orphacom/cahiers/docs/GB/list_of_orphan_drugs_in_europe.pdf, Accessed 5th March 2020.

12 Bolislis WR, Corriol-Rohou S, Hill-Venning C, Hoogland H, Joos A, King D, et al. Orphan Medicines for Pediatric Use: A Focus on the European Union. Clin Ther. 2019 Dec;41(12):2630-42.

13 Bolislis WR, Corriol-Rohou S, Hill-Venning C, Hoogland H, Joos A, King D, et al. Orphan Medicines for Pediatric Use: A Focus on the European Union. Clin Ther. 2019 Dec;41(12):2630-42.

14 Tambuyzer E. Rare diseases, orphan drugs and their regulation: questions and misconceptions. Nat Rev Drug Discov. 2010 Dec;9(12):921-9.

15 European Commission Report Conference "Medicines for Rare Diseases and Children: Learning from the Past, Looking to the Future". Brussels, June 2019.

16 Bolislis WR, Corriol-Rohou S, Hill-Venning C, Hoogland H, Joos A, King D, et al. Orphan Medicines for Pediatric Use: A Focus on the European Union. Clin Ther. 2019 Dec;41(12):2630-42.

17 Kanavos P, Sullivan R, Lewison G, Schurer W, Eckhouse S, Vlachopioti Z. The Role of Funding and Policies on Innovation in Cancer Drug Development. ecancer. 2010;4:164.

18 Vassal G, Kearns P, Blanc P, Scobie N, Heenen D, Pearson A. Orphan Drug Regulation: A missed opportunity for children and adolescents with cancer. Eur J Cancer. 2017 Oct;84:149-58.

19 European Medicines Agency. 10-year report to the European Commission: general report on the experience acquired as a result of the application of the paediatric regulation https://ec.europa.eu/health/sites/health/files/ files/paediatrics/docs/paediatrics_10_years_ema_technical_report.pdf (August 15, 2017). Accessed 5th Mar 2020.

20 Zamora B, Maignen F, O’Neill P, Mestre-Ferrandiz J, Garau M. Comparing access to orphan medicinal products in Europe. Orphanet J Rare Dis. 2019 May;14(1):95.

21 Bolislis WR, Corriol-Rohou S, Hill-Venning C, Hoogland H, Joos A, King D, et al. Orphan Medicines for Pediatric Use: A Focus on the European Union. Clin Ther. 2019 Dec;41(12):2630-42.

22 Guideline on aspects of the application of Article 8(1) and (3) of Regulation (EC) No 141/2000: Assessing similarity of medicinal products versus authorised orphan medicinal products benefiting from market exclusivity and applying derogations from that market exclusivity. Official Journal of the European Union. 2008/C $242 / 08$. 
23 Hall AK, Carlson MR. The current status of orphan drug development in Europe and the US. Intractable Rare Dis Res. 2014 Feb;3(1):1-7.

24 European Commission Report Conference "Medicines for Rare Diseases and Children: Learning from the Past, Looking to the Future". Brussels, June 2019.

25 Giannuzzi V, Landi A, Bosone E, et al. Failures to further developing orphan medicinal products after designation granted in Europe: an analysis of marketing authorisation failures and abandoned drugs. BMJ Open. 2017;7(9):e017358.

26 European Medicines Agency. Annual Report on the Use of the Special Contribution for Orphan Medicinal Products (January 31, 2019). https://www.ema.europa.eu/en/documents/report/annual-report-usespecial-contribution-orphan-medicinal-products-2018_en.pdf. Accessed 1th Nov 2019.

27 Copenhagen Economics. Study on the economic impact of supplementary protection certificates, pharmaceutical incentives and rewards in Europe. May 2018.

28 European Commission Report Conference "Medicines for Rare Diseases and Children: Learning from the Past, Looking to the Future". Brussels, June 2019

29 Westermark K, Holm BB, Söderholm M, Llinares-Garcia J, Rivière F, Aarum S, et al.; Committee for Orphan Medicinal Products and the European Medicines. European regulation on orphan medicinal products: 10 years of experience and future perspectives. Nat Rev Drug Discov. 2011 May;10(5):341-9.

30 Detiček A, Locatelli I, Kos M. Patient access to medicines for rare diseases in European countries. Value Health. 2018 May;21(5):553-60.

31 Bolislis WR, Corriol-Rohou S, Hill-Venning C, Hoogland H, Joos A, King D, et al. Orphan Medicines for Pediatric Use: A Focus on the European Union. Clin Ther. 2019 Dec;41(12):2630-42.

32 European Commission, 2016. Inventory of Union and Member State incentives to support research into, and the development and availability of, orphan medicinal products. State of play 2015.

33 Kawalec P, Sagan A, Pilc A. The correlation between HTA recommendations and reimbursement status of orphan drugs in Europe. Orphanet J Rare Dis. 2016 Sep;11(1):122.

34 Garau M, Mestre-Ferrandiz J. Access mechanisms for orphan drugs: a comparative study of selected European countries. 2009.

35 European Medicines Agency. Biosimilar medicines: marketing authorisation, Cited February 17, 2020. Available from: https://www.ema.europa.eu/en/human-regulatory/marketing-authorisation/biosimilarmedicines-marketing-authorisation\#1.-eligibility-and-reference-product-section.

36 PHARMIG Association of the Austrian Pharmaceutical Industry, Submission of feedback on the Roadmap for the Evaluation of the legislation on medicines for children and rare diseases (medicines for special populations). 8 January 2018. 